Editorial

\title{
P53 levels govern the choice between senescence and quiescence
}

\author{
David W. Meek \\ Biomedical Research Institute, University of Dundee, UK
}

Commentary on: OV Leontieva, AV Gudkov, and MV Blagosklonny. Weak p53 permits senescence during cell cycle arrest. Cell Cycle 2010; 9: in press

E-mail:_d.w.meek@dundee.ac.uk

The mechanism(s) by which p53 chooses between outcomes of senescence or quiescence has remained elusive. A paper published in the recent issue of Cell Cycle indicates that this decision may be determined by p53 levels and their ability to selectively downregulate $\mathrm{mTOR}$ signaling.

Activation of p53 plays a pivotal role in protecting against the survival and systemic spread of potential tumour cells. It can also influence the aging process and, reciprocally, p53 function has been reported to decline with age $[1,2]$.

The biological outcome of inducing p53 is of great topical interest, with much focus on the numerous factors that lead to growth arrest or programmed cell death (apoptosis). Until recently, however, less attention had been given to the mechanisms governing the choice between two distinct forms of growth arrest: quiescence, where cells enter a reversible non-cycling state, and senescence, an irreversible loss of proliferative potential that is coupled with specific changes in cell morphology and gene expression. However, fascinating insight into this issue has come from the work of Blagosklonny, Gudkov and colleagues, culminating in an article in Cell Cycle journal describing a role for increased p53 activity in favouring quiescence over senescence [3]. Significantly, while cellular senescence is of central relevance to the aging process, growing evidence that it may also be a key mechanism of tumour suppression [4] has generated much excitement over the last few years and underscores the importance of understanding its regulation.

Recent studies from the Blagosklonny and Gudkov groups have shown that inducible ectopic expression of p21, a key p53 downstream target that is required for both senescence and quiescence, drives HT1080 fibro- sarcoma cells into senescence [5]. Contrary to expectation, co-activation of p53 using the MDM2 inhibitor Nutlin-3a suppresses the senescence mediated by $\mathrm{p} 21$ and makes the cells quiescent without altering the levels of p21. Notably, rapamycin, a classical inhibitor of the mTOR pathway, can also suppress p21mediated senescence suggesting the possibility that p53 might supress senescence by inhibiting mTOR signalling. Several key components of the mTOR pathway are, in fact, down-regulated by p53 [6] and, consistent with this idea, the Blagosklonny and Gudkov labs showed, in a subsequent publication [7], that silencing the expression of TSC2, an upstream inhibitor of mTORC1 that is induced by p53, partially changed Nutlin 3a-induced quiescence into senescence. In a reciprocal manner, cell lines that preferentially go into senesence upon Nultin-3a treatment are shunted back into quiescence if rapamycin is co-administered [7].

Having defined a model in which the choice between senescence or quiescence can depend upon mTOR signaling, the collaborating groups addressed an earlier observation that some p53 inducers, such as the DNA damaging agent doxorubicin, promote senescence while others, such as Nutlin $3 \mathrm{a}$, lead to quiescence in the same cell type. Nutlin 3 a works simply by uncoupling p53 from its negative regulator MDM2. Doxorubicin, on the other hand, not only activates the DNA damage pathways but also promotes a series of phosphorylation and acetylation events in p53 that can fine tune p53 function. So do these DNA damage-related events somehow interfere with suppression of senescence by p53 or is it the case that different levels of p53 induction by the two different stimuli are responsible for the different outcomes? Working with immortalised human fibroblasts that undergo senescence upon treatment with doxorubicin, they now show [3] that low, but not high doses of this drug drive the cells into senescence. The finding that doxorubicin can bring 
about both outcomes, depending on dose, effectively rules out the notion that the DNA damage pathways themselves mediate the switch. Moreover, the observation that suppression of doxorubicin-mediated senescence by Nutlin-3a is accompanied by superelevated p53 levels favours the model that p53 levels are the deciding factor. Also consistent with this model, Nutlin-3a itself, when given at low doses, favours a senescent phenotype although, curiously, this is only a partial effect. Collectively, these analyses pinpoint p53mediated inhibition of the mTOR pathway as a major effector in suppressing senescence, depending on whether p53 levels are above or below a critical threshold.

These fascinating new findings also raise some new and pertinent questions. For example, how do different p53 levels govern the senescence/quiescence switch? Presumably differential p53 sensitivity is likely to play a key role and analysis of the expression levels of the various mTOR target genes and their respective promoter activites may provide further evidence to support the model. Additionally, how does the mTOR pathway promote senescence mechanistically? What other pathways may influence the sensecence/quiescence choice and why does extended Nutlin3a treatment lead to senescence [3]? What are the implications for tumour suppression and for aging? And what lessons may be learned from the use of Nutlin-3a? This is a powerful inducer of p53 which, even at low levels, does not appear to bring about full commitment to senescence [3]. Perhaps, however, it lacks the subtlety, provided possibly by post-translational modifications or other stimulus-dependent events, that is so exquisitely essential to fine-tuning the p53 response and governing its eventual biological outcome. Further investigation should provide some enlightening answers.

\section{REFERENCES}

1. Feng $Z$ et al. Declining p53 function in the aging process: $a$ possible mechanism for the increased p53 tumor incidence in older populations. Proc Nalt Acad Sci U S A 2007;104:1663316638.

2. Matheu $A$ et al. Delayed ageing through DNA damage protection by the Arf/p53 pathway. Nature 2007;448:375-379.

3. Leontieva OV, Gudkov AV and Blagosklonny MV. Weak p53 permits senescence during cell cycle arrest. Cell Cycle 2010; 9: in press.

4. Campisi J and d'Adda di Fagagna F. Cellular senescence: when bad things happen to good cells. Nat Rev Mol Cell Biol 2007;8:729-740.

5. Demidenko ZN, Korotchkina LG, Gudkov AV and Blagosklonny MV. Paradoxical suppression of cellular senescence by p53. Proc Natl Acad Sci U S A 2010;107:9660-9664.
6. Feng $Z$ and Levine AJ. The regulation of energy metabolism and the IGF-1/mTOR pathways by the p53 protein. Trends Cell Biol 2010;20:427-434.

7. Korotchkina LG, Leontieva OV, Bukreeva El, Demidenko ZN, Gudkov AV and Blagosklonny MV. The choice between p53induced senescence and quiescence is determined in part by the mTOR pathway. Aging 2010;2:344-352. 